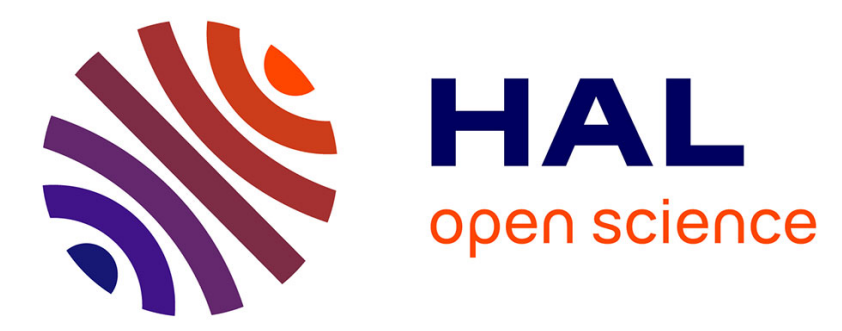

\title{
Heterogeneous crystallization near structured walls in quenched two-dimensional binary colloidal suspensions
}

\author{
Lahcen Assoud, Rene Messina, Hartmut Loewen
}

\section{To cite this version:}

Lahcen Assoud, Rene Messina, Hartmut Loewen. Heterogeneous crystallization near structured walls in quenched two-dimensional binary colloidal suspensions. Molecular Physics, 2011, 109 (07-10), pp.1385-1391. 10.1080/00268976.2011.562871 . hal-00692131

\section{HAL Id: hal-00692131 \\ https://hal.science/hal-00692131}

Submitted on 28 Apr 2012

HAL is a multi-disciplinary open access archive for the deposit and dissemination of scientific research documents, whether they are published or not. The documents may come from teaching and research institutions in France or abroad, or from public or private research centers.
L'archive ouverte pluridisciplinaire HAL, est destinée au dépôt et à la diffusion de documents scientifiques de niveau recherche, publiés ou non, émanant des établissements d'enseignement et de recherche français ou étrangers, des laboratoires publics ou privés. 


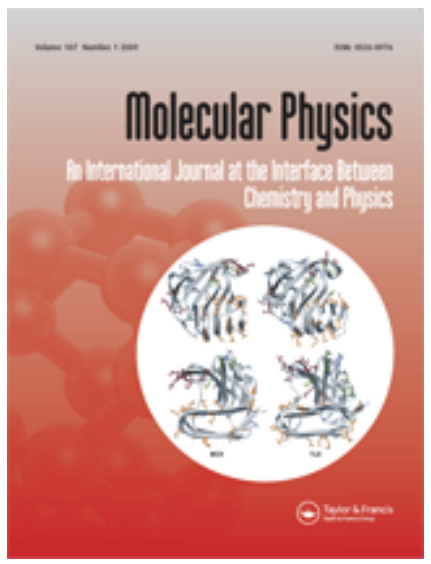

\section{Heterogeneous crystallization near structured walls in quenched two-dimensional binary colloidal suspensions}

\begin{tabular}{|c|c|}
\hline Journal: & Molecular Physics \\
\hline Manuscript ID: & TMPH-2010-0431.R1 \\
\hline Manuscript Type: & Special Issue paper - In honour of Bob Evans \\
\hline $\begin{array}{r}\text { Date Submitted by the } \\
\text { Author: }\end{array}$ & 03-Feb-2011 \\
\hline Complete List of Authors: & $\begin{array}{l}\text { Assoud, Lahcen; Heinrich-Heine-Universitaet } \\
\text { Messina, Rene; Heinrich-Heine-Universitaet Duesseldorf, Institut } \\
\text { fuer Theoretische Physik II } \\
\text { Loewen, Hartmut; Heinrich-Heine-Universitaet }\end{array}$ \\
\hline Keywords: & $\begin{array}{l}\text { Heterogeneous crystallization, nucleation, colloids, binary mixtures, } \\
\text { Brownian dynamics }\end{array}$ \\
\hline \multicolumn{2}{|c|}{$\begin{array}{l}\text { Note: The following files were submitted by the author for peer review, but cannot be converted } \\
\text { to PDF. You must view these files (e.g. movies) online. }\end{array}$} \\
\hline \multicolumn{2}{|c|}{$\begin{array}{l}\text { Assoud_Het_Nuc.bib } \\
\text { Assoud_Het_Nuc_molphys_RESUB.tex } \\
\text { Assoud_Het_Nuc_molphys_RESUB.bbl }\end{array}$} \\
\hline
\end{tabular}

\section{SCHOLARONE Manuscripts}


René Messina

Institut für Theoretische Physik II

Heinrich-Heine-Universität Düsseldorf

Universitätsstrasse 1

D-40225 Düsseldorf

Germany

email: messina@thphy.uni-duesseldorf.de

Tel: $(+49)$ 211-8115874

Fax: $(+49) 211-8112262$

February 3, 2011

Electronic resubmission to Molecular Physics

Manuscript Title: Heterogeneous crystallization near structured walls in quenched twodimensional binary colloidal suspensions

Your Ref: TMPH-2010-0431

Dear Prof. J.P. Hansen,

please find enclosed a revised version of the manuscript, as well as our reply to the Referees comments. We believe that we have cleared out the points raised by the Referees and hope that the paper is now suitable for publication in Molecular Physics.

Sincerely yours,

René Messina 


\section{Reply to Referee 1}

We thank the Referee for his/her constructive and critical remarks which have led to considerable improvements of the manuscript. In detail our reply is as follows.

1. It may be helpful for readers to have the equilibrium phase diagram of the system.

We have carefully discussed this point among the authors and have concluded that it is not that instructive to add the full (two-dimensional) phase diagram, given the fact that in the present study only a single point is considered, namely the $\mathrm{S}(\mathrm{AB})$ phase. Note that the full phase diagram contains 17 phases and it may therefore obscure the simple situation we consider here. Of course we mentioned here the corresponding reference 34.

2. The actual method realizing a quench to $\Gamma=\infty$ (presumably, switching off the thermal noises) is more explicitly described.

We have taken this relevant remark into account and added the following sentence part (see page 3 ):

".. $\Gamma=\infty$ (i.e., the temperature is set to zero leading to a steepest descent quench)".

3. I recommend the authors to show plots of the fraction of $A$ particle in square (or triangular) crystallites at $\Gamma=\infty$ as a function of $\Gamma_{\text {int }}$. It is not so easy to figure out the dependence of the fraction of $A$ particles in triangular crystallites as a function of $\Gamma_{\text {int }}$ in Fig. 2(b).

The Referee's request was exactly contained in Fig 3 (see black lines there). Since this feature can be overlooked, we have made the connection clearer in the text (see page 4): "This latter feature is precisely addressed in Fig. 3."

4. It may be better to explicitly mention that the faster motion of big A particles near wall $A$ is due to the much stronger dipolar interaction between AA pairs. In this sense, the kinetic selection near wall 2 seems to reflect the difference in the interaction strength between $A A$ and $A B$. This implies that some caution is necessary for the usage of the terms, kinetics and energetics.

We agree with the Referee that the usage of the terms "kinetics" and "energetics" was not clearly physically motivated. To remedy this, we have now removed the misleading sentence in the abstract containing kinetics and energetics. Moreover, the term "kinetics" is physically explained in line with the Referee's suggestion. Thereby we have added the following explanation in the text, see page 2 :

“..., mainly governed by the much stronger $A A$ interaction."

5. In the abstract the authors stated "the number of triangular crystallites close to the wall which has outermost fixed small particles is significantly higher than in the unconfined case". However, this looks odd and contradicts with the results shown in Fig. 2(b). Similarly, in page 5, the right column, 4th line, "wall 2" may be "wall 1".

Indeed there is a mistake in the abstract, and one should read "fixed big particles".

In page 5 , the right column, $6 \mathrm{Th}$ line, "wall 2 " is correct so. 
6. There are also some typos. In the abstract "It found" should be replaced by "It is found". In the caption of Fig. 2, "to to" should be replaced by "to". Fixed. 


\section{Reply to Referee 2}

We thank the Referee for his/her constructive remarks which have led to considerable improvements of the manuscript. In detail, our reply is as follows.

- The study has been done with a fixed number of particles (2x900), and averages have been done over 10 runs. The authors mention that the large scatter in the data may be due to the small amount of averages. It would improve the manuscript further to see the effect of the number of particles on the observed phenomena and/or the effect of the number of averages on the smoothness of the data.

We agree with the Referee that the data would be smoother by increasing even more the number of particles and/or realizations. However, given the already enormous computational time devoted to this study, a neat improving of the statistics is not achievable within the given time constraint.

- In the caption of Figure 1 both different wall structures are labeled "wall 1". Most likely the authors have in mind to label the right wall by "wall 2 ".

Fixed.

- In the abstract, line 22, the sentence starting with "It found" looks a bit strange, is there perhaps an "is" missing?

Fixed. 


\section{INTRODUCTION}

In recent years, research has focused on the dynamics of crystal formation from an undercooled melt in order to identify the underlying molecular processes. This is important both from a fundamental point of view but also for many applications ranging from metallurgy [1], material science (e.g. for the formation of optical bandgap crystals) [2] to biology (e.g. for protein crystallization) [3-5]. In particular, real space information arising from computer simulations [6-10], density functional approaches $[11,12]$ of model systems, phase field crystal calculations [13-16] and real-space experiments on colloidal dispersions [17-19] have elucidated the kinetic pathways of crystal nucleation and subsequent microstructure formation on the particle scale. Though more relevant for practical applications, heterogeneous crystal nucleation, which is initiated at impurities or system boundaries, is much less understood than homogeneous crystal nucleation in a bulk fluid [20, 21]. Heterogeneous nucleation was studied for one-component systems (e.g. hard spheres, two-dimensional dipoles) near smooth [7, 22] or structured [23-26] walls, near external seed particles [27-29] or at imposed nucleation clusters [12]. However, quantitative and systematic studies on heterogeneous nucleation in two-component (binary) systems are sparse [30]. Binary system have much more freedom for equilibrium crystal structures and therefore the heterogeneous crystallization is expected to be much richer: it depends critically on the thermodynamic parameters characterizing the bulk system and the structure of the external seed.

In this paper, we study heterogeneous crystallization near patterned walls in a two-dimensional model for binary dipolar colloidal suspensions. The particles interact via pairwise forces deriving from a repulsive potential of two parallel dipoles. This model is realized for superparamagnetic colloidal particles at a pending air-water interface in an external magnetic field which tunes the dipole moments [31-33] and the two-dimensional phase behavior at zero temperature are known [34]. The formation of crystallites after a sudden increase of the field (corresponding to a fast temperature quench) was explored in real-space experiments and compared to Brownian dynamics computer simulations [35]. Thereby, the formation of crystalline patches with different structures corresponding to stable and metastable phases was monitored as a function of time [35]. As a result, two-dimensional homogeneous crystallization after a fast quench exhibited a complex structure formation which involves disordered parts and crystallites. Apart from the thermodynamically stable structure, also metastable structures (such as triangles of big particles) were emerging as well [35]. On the time-scale explored by the experiment and the computer simulation, the thermodynamically stable crystalline state was not reached [35]. It is tempting to generalize these studies to heterogeneous crystallization since the (wall-free) behaviour is well characterized and 
can be used as a reference for comparison.

Here we study heterogeneous crystallization near patterned walls by extensive Brownian dynamics computer simulations. A similar setup for a one-component twodimensional system was studied in [36]. The wall pattern exactly corresponds to the equilibrium crystal structure after the quench which is an alternating binary equimolar square crystal $S(A B)$. Two different wall patterns are considered which are composed of fixed particles forming a stripe of the stable square crystal. In principle, this can be realized experimentally by using optical tweezers. The walls are a cut through the $S(A B)$ crystal along the crystallographic (10)-direction where the outermost layer of fixed particle is either a row of big or small particles. (By "big" or "small" we refer to particles with a big or small dipole moment.) Since in both cases the wall structure imprints locally the equilibrium crystal, one would expect after a quench that heterogeneous crystallization near the walls is favored and that crystallites with a metastable structure should be suppressed - relative to the unconfined case - at the walls. Contrarily we find that crystalline patches which involve big particles in a triangular structure are favored near the wall with fixed outermost big particles. The concentration of big particles with a triangular crystalline neighborhood is even larger than in the unconfined case. We attribute this unusual finding to pure kinetics of particle rearrangements after the quench, mainly governed by the much stronger $A A$ interaction. It is not driven by energetics and therefore not simply contained in traditional classical nucleation theory $[37,38]$.

In principle, our simulation results can be verified in real-space microscopy experiments of two-dimensional superparamagnetic particles [39-43] see also [44, 45] for alternate set-ups. The immobile particles building the external walls can be fixed by optical tweezers. Apart from that our results give more general insight into the molecular (i.e. particle-resolved) processes of heterogeneous crystallization, in particular at very fast and deep quenches where it competes with glass formation. Our findings demonstrate that the emergent behaviour is not controlled by equilibrium-like concepts (like line tensions) which form the basics of classical nucleation theory [46] but may be governed by local kinetics. This is expected to hold in a more general sense for any binary system when quenched quickly into a low-temperature state [47]. Our findings are also relevant for cooling-rate dependence of vitrification which competes with crystallization [10].

The paper is organized as follows: in section II, we describe the model and the simulation technique employed. In section III, we summarize the results and present snapshots and statistically averaged quantities after various quenches which reveal the heterogeneous crystallization behaviour. We devote chapter IV to a discussion and conclude in section $\mathrm{V}$.

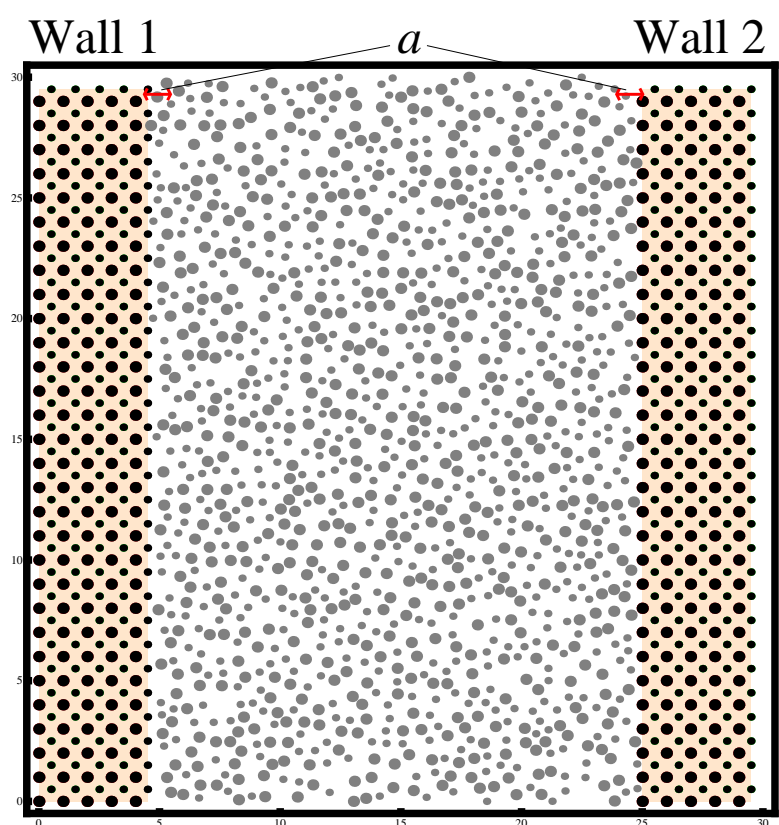

FIG. 1: Schematic illustration of colloidal particles confined between two walls at $\Gamma_{\text {init }}=0$ with a finite particle wall interaction. The big circles (small) represent A-particles (Bparticles). The fixed particles are indicated by black full circles and are backed by orange ground. All other large particles are shown in grey. The distance $a$ represents the average interparticle separation between big particles. The two different wall structures are referred to as wall 1 (left wall, where the outermost layer consists of fixed small particles) and wall 1 (right wall, where the outermost layer consists of fixed big particles)

\section{MODEL}

The system consists of a suspension of two species of point-like super-paramagnetic colloidal particles denoted by $A$ and $B$ which are confined to a two-dimensional planar interface. Those particles are characterized by different magnetic dipole moments $m_{A}$ and $m_{B}$, where $m=m_{B} / m_{A}$ is the dipole-strength ratio. The dipoles are induced by an external magnetic field $H$ as $m_{i}=\chi_{i} H$ $(i=A, B)$ where $\chi_{i}$ denotes the magnetic susceptibility. The magnetic field is applied perpendicular to the two-dimensional interface containing the particles. In the following the dipole-strength ratio $m$ is fixed to 0.1 corresponding to recent experimental samples [35, 41, 42]. The relative composition $X=N_{B} /\left(N_{A}+N_{B}\right)$ of $B$ particles is fixed at $50 \%$, hence we are considering an equimolar mixture. The particles interact via a repulsive pair potential of two parallel dipoles of the form

$$
u_{i j}(r)=\frac{\mu_{0}}{4 \pi} m_{i} m_{j} / r^{3}=\frac{\mu_{0}}{4 \pi} \chi_{i} \chi_{j} H^{2} / r^{3} \quad(i, j=A, B) .
$$


where $r$ denotes the distance between two particles. For this inverse power potential, at fixed composition $X$ and susceptibility ratio $\chi_{B} / \chi_{A}$, all static quantities depend solely [48] on a dimensionless interaction strength (or coupling constant)

$$
\Gamma=\frac{\mu_{0}}{4 \pi} \frac{\chi_{A}^{2} H^{2}}{k_{B} T a^{3}}
$$

where $k_{B} T$ is the thermal energy and $a=1 / \sqrt{\rho_{A}}$ the average interparticle separation between $A$ particles. Here, $\rho_{A}$ denotes the areal number density of the $A$ particles.

We perform non-equilibrium Brownian dynamics (BD) computer simulations [49, 50]. Hydrodynamic interactions are neglected. The Brownian time scale is set by the short-time diffusion constant $D_{A}$ of the $A$ particles. Knowing that this diffusion constant scales with the inverse of the radius of a particle, $D_{B}$ was chosen such that $D_{B} / D_{A}=1.7$ corresponding to the physical diameter ratio of the experimental samples [35, 41, 42]. A finite time step $\delta t=10^{-5} \tau$, where $\tau=a^{2} / D_{A}$, was used in the simulations.

Two different wall patterns consist of fixed particles belonging to a crystalline centered square structure. These walls are chosen with a finite width of $20 a$ and periodic boundary conditions are applied in both directions. A simulation box set-up is sketched in Figure 1. The blackcoded particles indicate the fixed $A$ and $B$ particles which belong to square clusters. The outermost row of wall 1 (left wall in Figure 1) is composed of small particles. On the other hand, big particles form the outermost row of wall 2 (right wall in Figure 1). $N_{A}=900 A$ particles and $N_{B}=900 B$ particles were placed in a square box including the fixed wall particles. As a reference, we have also performed simulations in a square simulation box with periodic boundary conditions in the absence of any walls with the same number of particles.

Starting from an initial configuration of noninteracting particles which initially do not penetrate the walls (see the snapshot in Figure 1) we have changed the coupling $\Gamma$ as a function of time by changing the magnetic field (while keeping the densities and the temperature fixed). This corresponds formally to a cooling process. The cooling history is as follows: first we increase the interaction strength $\Gamma$ by constant steps of $d \Gamma=5$ and within a time $\Delta t=1 \tau$ to let the system equilibrate. This procedure is continued until a prescribed coupling $\Gamma_{\text {init }}$ (initial coupling) is reached. From this configuration, a sudden quench is performed to $\Gamma=\infty$ (i.e., the temperature is set to zero leading to a steepest descent quench). The time for the instantaneous quench sets the zero on our time axis. We checked that the initial state with $\Gamma=\Gamma_{\text {init }}$ was equilibrated enough. Details of the cooling history are shown in Figure 2. Finally we averaged over 10 different starting configurations in order to reduce the statistical error.

Our aim is to study crystal nucleation near the walls after the sudden quench. Therefore we have used criteria to define $A$ particles which have a pure triangu-
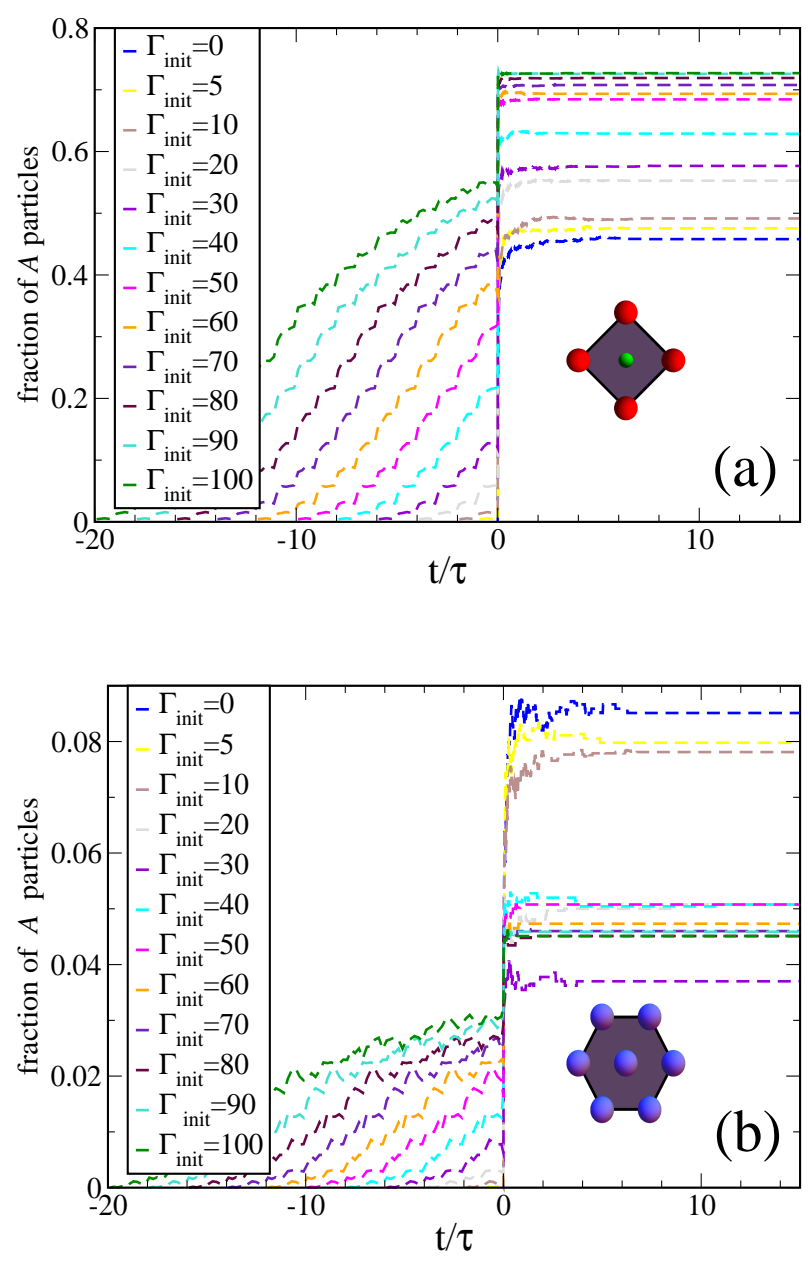

FIG. 2: (a) Fraction of $A$ particles belonging to a crystalline square (see inset) and (b) fraction of $A$ particles belonging to a crystalline triangular surrounding (see inset) versus reduced time $t / \tau$ for instantaneous "steepest descent" quench from different $\Gamma_{\text {init }}$ to $\Gamma=\infty$ in the unconfined case. For negative times, the system is slowly cooled until reach $\Gamma_{\text {init }}$ for $t=0$. Details of the stepwise cooling history are given in the text. Then the system is instantaneously brought to $\Gamma=\infty$ at $t=0$. Different colors correspond to different $\Gamma_{\text {init }}$, see legend.

lar surrounding of other $A$ particles, i.e. which are close to a cut-out of a pure triangular $A$ crystal, and, likewise, we have identified $A$ and $B$ particles which form locally an equimolar square lattice $S(A B)$. The corresponding two structure elements are shown as insets in Figure 2. Following the definition used in Refs. [41], in detail, we associate a triangular surrounding to an $A$ particle if the following two criteria are fulfilled simultaneously: (i) The 6 -fold bond order parameter $p_{6}=\sqrt{\Psi_{6}^{*} \Psi_{6}}$ (where $\Psi_{6}=\frac{1}{6} \sum_{N N}^{6} \exp \left(i 6 \theta_{N N}\right)$ with $\theta_{N N}$ denoting the angles of the six nearest neighbour bonds relative to a fixed reference) is larger than 0.94. (ii) The relative bond length deviation $b_{6}=\frac{1}{6} \sum_{N N}^{6} \frac{\left|l_{N N}-\bar{l}\right|}{\bar{l}}$ where $\bar{l}$ is the average length of the six bond lengths $l_{N N}$ is 
smaller than 0.04. This double condition selects local configurations close to those of a perfect triangular lattice where $p_{6}$ is unity and $b_{6}$ vanishes. Likewise we define a square surrounding around a $B$ particle by the criteria: (i) The 4 -fold bond order parameter $p_{4}=\sqrt{\Psi_{4}^{*} \Psi_{4}}$ (where $\Psi_{4}=\frac{1}{4} \sum_{N N}^{4} \exp \left(i 4 \theta_{N N}\right)$ with $\theta_{N N}$ denoting the bond angles of the four nearest neighbour $A B$ bonds) is larger than 0.92 . (ii) The corresponding relative $A B$ bond length deviation $b_{4}$ is smaller than 0.05 .

\section{RESULTS}

Figures 2(a) and 2(b) refer to the unconfined case in the absence of walls. The fraction of $A$ particles with a triangular and with a square surrounding as a function of time is displayed, respectively, for different $\Gamma_{\text {init }}$. The average here is over all mobile $A$ particles of the system. First the cooling preparation can be seen, shown for negative times in Figure 2. The particle number is increasing with time as the coupling is stepwise increased. After each increase the system equilibrates. This has been checked by taking slower cooling rates where relaxation time after each cooling step is doubled which did not affect the data. Hence, at different $\Gamma_{\text {init }}$, the system is preordered containing already a small amount of crystallites with square and triangular local ordering. After the sudden quench (performed at $t=0$ ), the number of crystalline particles is steeply increasing first and then saturates on the time-scale of $15 \tau$ explored by the simulation. This latter feature is precisely addressed in Fig. 3. In general there are less particles with triangular order than those with square order (see the different scales in Figure 2(a) and 2(b)). Therefore the data on the triangular particles are more noisy in general.

In general, there is an opposite trend of the number of square and triangular crystallites with $\Gamma_{\text {init }}$ : while the number of square particles is growing with $\Gamma_{\text {init }}$, the number of triangular particles is decreasing with $\Gamma_{\text {init }}$. This has to do with the fact that there is pre-ordering in the fluid state which corresponds to the thermodynamically stable ground state $S(A B)$. Hence an equilibrated highly correlated fluid state is quenched more easily into crystalline patches with square-like symmetry. Conversely, the triangular structure is occurring most in uncorrelated systems upon a sudden cooling process. However, there is a minimal number of triangular particles for $\Gamma_{\text {init }}=30$ which possibly is within the statistical accuracy (see also data for the unconfined case in Figure $3 \mathrm{~b}$ ).

We now address the question of how the walls influence the local occurrence of crystallites relative to their formation in the unconfined case. Reference data with the same cooling history but in a system without any walls are produced similar to that shown in Ref. [35]. In order to monitor the crystallites close to the walls we have defined a strip of width $a$ parallel to wall and count only particles within this strip. The relative fraction of $A$-particles with triangular or square ordering within this
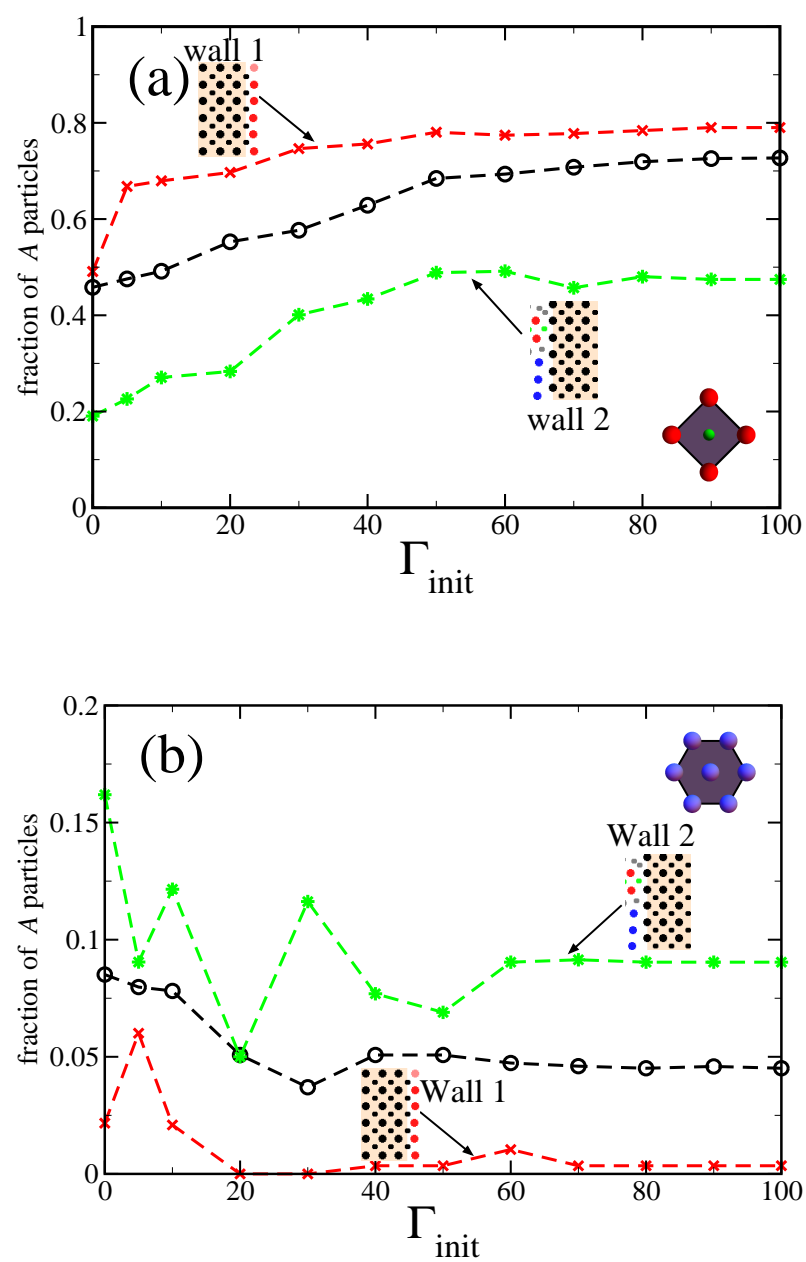

FIG. 3: (a) Fraction of $A$ particles belonging to a crystalline square surrounding (see inset) and (b) fraction of $A$ particles belonging to a crystalline triangular surrounding (see inset) versus $\Gamma_{\text {init }} 15 \tau$ after the quench. Red line: within a stripe of width $a$ near wall 1 , green line: within a stripe of width $a$ near wall 2 , black line: in the unconfined case (as a reference).

strip indicates the amount of heterogeneous crystallization near the walls. This quantity is shown in Figures $3(\mathrm{a})$ and $3(\mathrm{~b})$ as a function of the initial coupling $\Gamma_{\text {init }}$ $15 \tau$ after the quench together with the wall-free data as a reference. As expected, the fraction of particles with a square-like surrounding depicted in Figure 3(a) is increasing with $\Gamma_{\text {init }}$. Close to wall 1 , there are more such particles than in the unconfined case which is understood by the fact that the wall 1 induces square like ordering into the adjacent fluid mixture. However, although wall 2 is also imprinting the same square-like structure into the fluid, there are fewer of these particles at the wall 2 than in the unconfined case. This is most pronounced for small $\Gamma_{\text {init }}$ but still significant at high $\Gamma_{\text {init }}$. Conversely, the number of triangular particles at wall 1 is much lower than in the unconfined case while at wall 2 this number is definitively higher than in the unconfined case, see Figure 
3(b). The large fluctuations have to do with the limited set of 10 different initial configurations used for the average. Still the absolute fraction is such that there are more square-like particles than triangular-like particles (compare the different scales of Figures 3(a) and 3(b)). It is only for wall 2 at low $\Gamma_{\text {init }}$ that these two numbers are comparable.

Actual particle snapshots after the quench are shown in figure 4 for $\Gamma_{\text {init }}=0$ and $\Gamma_{\text {init }}=100$ with appropriately color-coded particles indicating the locations of $\mathrm{A}$ and $\mathrm{B}$ particles which belong to local triangular and square clusters. If an A particle has a triangular surrounding, all seven A particles including the central one with its full surrounding are shown in blue. Conversely, if a $\mathrm{B}$ particle has a square surrounding, it is colored in green and its four A neighbors are colored in red. All particles which belong both to the blue and the red class are shown in pink. In consistence with Figure 2, the crystalline clusters grow as a function of $\Gamma_{\text {init }}$. What can be deduced form the snapshots is that the actual particles clusters are small. On the time scale of the simulation they are fluctuating a bit, but are no significantly growing in size. Hence on the observation time-scale the system is far away from the thermodynamically stable state of a perfect square crystal between the two walls.

\section{DISCUSSION}

In the unconfined case, the stable state (or ground state) after a steepest descent quench is the stable crystalline lattice which minimizes the total potential energy at fixed composition. In our previous work [34], it was shown that the stable state is a square $S(A B)$ crystal which exactly coincides with the boundaries assumed in our simulation. Therefore the interfacial energy between this (wall-free) phase and the imposed boundary is zero. Moreover the spacing between the two wall is allowing an exact match of the $S(A B)$ crystal. This implies that the stable confined state is a strip (cutout) of the $S(A B)$ crystal. Hence the exact state is known and, given enough time and enough fluctuations, the system should reach this state after the quench. Moreover, the fixed boundaries in fact favor locally a (10)-oriented $S(A B)$ crystal. Therefore one expects that near the walls the $S(A B)$ crystalline structure is enhanced relative to its occurrence in the unconfined case, i.e. that the $S(A B)$ crystal will heterogeneously nucleate at the walls.

Strikingly, after a sudden quench, as realized in our simulations, the systems does not relax into the true ground state and even avoids the preferential $S(A B)$ structure near wall 2 where the outermost layer are fixed big (A) particles. Instead, locally a triangular crystal of pure $A$ particles is preferentially nucleated near wall 2 (even compared to the wall-free case as a reference). Near wall 1 , on the other hand, the behaviour is more like expected: here, more square-like $A$ particle are showing up than in the unconfined case pointing to the locally pre-
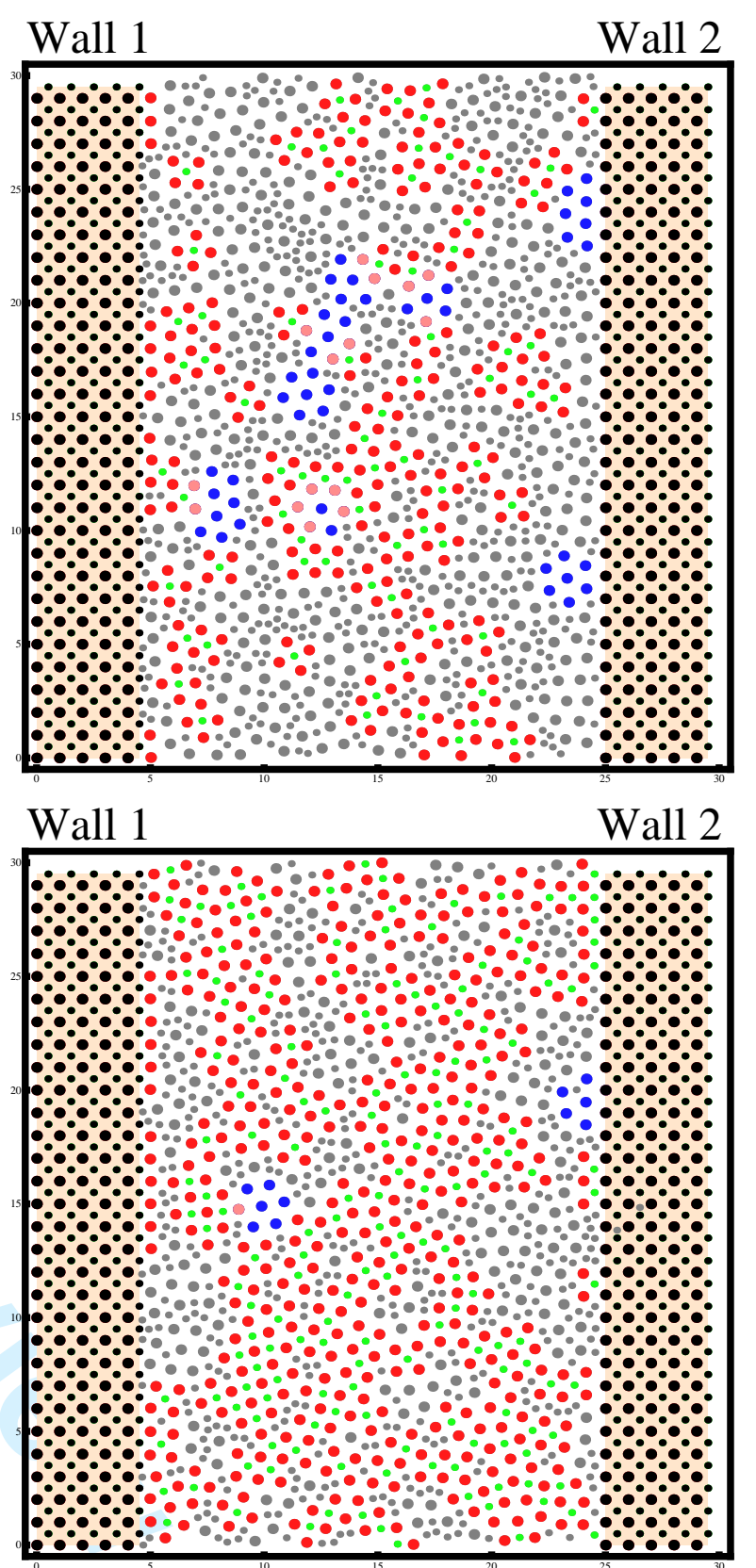

FIG. 4: Simulation snapshots $15 \tau$ after the instantaneous quenching process for (a) $\Gamma_{\text {init }}=0$ (b) $\Gamma_{\text {init }}=100$. Big particles are shown in blue if they belong to a triangular surrounding and in red if the belong to a square surrounding. The black full circles indicate fixed particles and all other large particles are shown in grey.

ferred $S(A B)$ crystal. The counter-intuitive behaviour near wall 2 has to do with the very fast quench. A simple argument goes along the following line: for a sudden quench first the big $A$ particle relax near a wall which is made by fixed $A$ particles only. Therefore, neglecting all small particles in the fast process of reordering, the 
big particles try to arrange in a triangular cluster and freeze in. Near wall 2, on the other hand, there is a finestructure of the outermost fixed small particles which locally forbids a triangular structure of the big particles. Clearly, classical nucleation theory which is formulated in terms of interfacial tensions would not predict the nucleation of triangular particles near wall 2. The failure of classical nucleation theory $[37,38]$ has to do with the rapid quench which violates the inherent conditions of local thermodynamic equilibrium.

However, the general scenario is compatible with the traditional Ostwald's step rule [51-53] of homogeneous nucleation which tells that if an unstable phase $\mathcal{A}$ transforms into a stable phase $\mathcal{B}$, a metastable phase $\mathcal{C}$ can intervene if it is "kinetically more accessible" than phase $\mathcal{B}$. In many (but not all) applications of the Ostwald's step rule a finite sheet of the metastable phase $\mathcal{C}$ even remains in equilibrium coexistence between phases $\mathcal{A}$ and $\mathcal{B}$. In this case, the equilibrium interfacial tension between $\mathcal{A}$ and $\mathcal{B}$ is minimized via the intervening layer of $\mathcal{C}$. In our case, the phases $\mathcal{A}$ and $\mathcal{B}$ are identical and hence an intervening layer in equilibrium is prohibited. Hence the occurrence of the triangular crystallites is completely due to kinetics.

\section{CONCLUSION}

In conclusion, we have studied heterogeneous crystallization near patterned walls by extensive Brownian dynamics computer simulations. The wall pattern corresponds exactly to the equilibrium crystal structure after the quench which is an alternating binary equimolar square crystal $S(A B)$. Two different wall patterns are considered (wall 1 and wall 2) which are made up by fixed particles forming a stripe of the stable square crystal. Since in both cases the wall structure imprints locally the equilibrium crystal, one would expect after a quench that heterogeneous crystallization near the walls is favored and that crystallites with a metastable structure should be suppressed - relative to the unconfined case at the walls. Contrarily we find that crystalline patches which involve big particles in a triangular structure are favored near the wall 2 with fixed outermost big particles even relative to the unconfined case. We attribute this to pure kinetics of particle rearrangements after the rapid quench. It is therefore not contained in traditional classical nucleation theory [37, 38].

In principle, our set-up studied can be realized experimentally by using optical tweezers in order to fix the wall particles. Real-space microscopy experiments of twodimensional superparamagnetic particles are quite common for binary mixtures and have been used to study the dynamics after a quench into the glass [39-43]. Our results provide also more general insight into the molecular (i.e. particle-resolved) processes of heterogeneous crystallization, in particular at very fast and deep quenches where it competes with glass formation. In fact, our findings demonstrate that the emergent behaviour is not controlled by equilibrium-like concepts (like line tensions) which form the basics of classical nucleation theory [46] but may be governed by local kinetics. This is expected to hold in a more general sense for any binary system when quenched quickly into a low-temperature state [47]. Our findings are therefore also relevant for the general relation between crystallization and vitrification [54-56].

\section{Acknowledgments}

We dedicate this paper to R. Evans who is one of the pioneers of statistical mechanics of confined systems. This work was financially supported by the DFG within SPP 1296.
[1] H. Emmerich, K. Binder, and B. Nestler, Phil. Mag. Lett. 87, 791 (2007).

[2] N. V. Dziomkina and G. J. Vancso, Soft Matter 1, 265 (2005).

[3] M. C. Wiener, Methods 34, 364 (2004).

[4] E. H. Snell and J. R. Helliwell, Rep. Prog. Phys. 68, 799 (2005).

[5] R. Sear, J. Phys.: Condens. Matter 19, 033101 (2007).

[6] S. Auer and D. Frenkel, Adv. Polym. Sci 173, 149 (2005).

[7] A. Cacciuto and D. Frenkel, Phys. Rev. E 72, 041604 (2005).

[8] C. Desgranges and J. Delhommelle, J. Chem. Phys. 126, 054501 (2007).

[9] T. Schilling et al., Phys. Rev. Lett. 105, 025701 (2010).

[10] H. Shintani and H. Tanaka, Nature Phys. 2, 200 (2006).

[11] R. Ohnesorge, H. Löwen, and H. Wagner, Phys. Rev. A 43, 2870 (1991).

[12] S. van Teeffelen, C. N. Likos, and H. Löwen, Phy. Rev. Lett. 100, 108302 (2008).
[13] H. Emmerich, Adv. Phys. 57, 1 (2008).

[14] L. Gránásy, T. Pusztai, and J. A. Warren, J. Phys.: Condens. Matter 16, R1204 (2004).

[15] L. Gránásy et al., J. Mater. Res. 21, 309 (2006).

[16] L. Gránásy, T. Pusztai, D. Saylor, and J. A. Warren, Phys. Rev. Lett. 98, 035703 (2007).

[17] U. Gasser, J. Phys.: Condens. Matter 21, 203101 (2009).

[18] U. Gasser et al., Science 292, 258 (2001).

[19] C. P. Royall, S. R. Williams, T. Ohtsuka, and H. Tanaka, Nature Materials 7, 556 (2008).

[20] H. Emmerich, J. Phys.: Condensed Matter 21, 460301 (2009).

[21] G. Kahl and H. Löwen, J. Phys.: Condensed Matter 21, 464101 (2009).

[22] P. Wette et al., J. Phys.: Condensed Matter 21, 464115 (2009).

[23] M. Heni and H. Löwen, Phys. Rev. Lett. 85, 3668 (2000).

[24] M. Heni and H. Löwen, J. Phys.: Condensed Matter 13, 4675 (2001). 
[25] W. S. Xu, Z. Y. Sun, and L. J. An, J. Chem. Phys. 132, 144506 (2010).

[26] S. van Teeffelen, H. Löwen, R. Backofen, and A. Voigt, Phys. Rev. E 79, 051404 (2009).

[27] V. W. A. de Villeneuve et al., Science 309, 1231 (2005).

[28] V. W. A. de Villeneuve et al., J. Phys.: Condens. Matter 17, S3371 (2005).

[29] A. Cacciuto, S. Auer, and D. Frenkel, Nature 428, 404 (2004).

[30] S. R. Williams, C. Royall, and G. Bryant, Phys. Rev. Lett. 100, 255502 (2008).

[31] N. Hoffmann et al., Phys. Rev. Lett. 97, 078301 (2006).

[32] L. Assoud et al., J. Phys.: Condensed Matter 21, 464114 (2009).

[33] Y. Terada and M. Tokuyama, J. Phys. Soc. Jpn. 79, $034802(2010)$.

[34] L. Assoud, R. Messina, and H. Löwen, Europhys. Letters 80, 48001 (2007).

[35] L. Assoud et al., Phys. Rev. Lett. 102, 238301 (2009).

[36] K. Franzrahe et al., J. Phys.: Condens. Matter 120, 404218 (2008).

[37] T. Young, Trans. Roy. Soc. London 95, 65 (1805).

[38] R. W. Cahn and P. Haasen, Physical Metallurgy (North Holland, Amsterdam, 1983)

[39] M. Kollmann et al., Europhys. Lett. 58, 919 (2002).

[40] H. König, R. Hund, K. Zahn, and G. Maret, Eur. Phys. J. E 18, 287 (2005).

[41] F. Ebert, P. Keim, and G. Maret, Eur. Phys. J. E 26, $161(2008)$.
[42] S. Mazoyer, F. Ebert, G. Maret, and P. Keim, Europhys. Lett. 88, 66004 (2009).

[43] F. Ebert, G. Maret, and P. Keim, Eur. Phys. J. E 29, 311 (2009).

[44] A. Pertsinidis and X. S. Ling, Nature 413, 147 (2001).

[45] Z. R. Wang, A. M. Alsayed, A. G. Yodh, and Y. L. Han, J. Chem. Phys. 132, 154501 (2010).

[46] L. Granasy and P. F. James, J. Non-Cryst. Solids 253, 210 (1999).

[47] V. A. Shneidman and D. R. Uhlmann, J. Chem. Phys. 108, 1094 (1998).

[48] J.-P. Hansen and I. R. MacDonald, Theory of Simple Liquids (Academic, New York, 1986), 2nd ed.

[49] M. P. Allen and D. J. Tildesley, Computer Simulation of Liquids (Oxford Science Publications, New York, 1987).

[50] For an equilibrium molecular dynamics simulation for a similar model, see T. Stirner, and J. Sun, Langmuir 21, 6636 (2005).

[51] W. Ostwald, Z. Phys. Chem. 22, 289 (1897).

[52] J. Bechhoefer, H. Löwen, and L. S. Tuckerman, Phys. Rev. Lett. 67, 1266 (1991).

[53] P. R. ten Wolde and D. Frenkel, Phys. Chem. Chem. Phys. 1, 2191 (1999).

[54] T. Kawasaki and H. Tanaka, Proc Natl Acad Sci USA 107, 14036 (2010).

[55] E. Zaccarelli et al., Phy. Rev. Lett. 103, 135704 (2009).

[56] T. Palberg, A. Stipp, and E. Bartsch, Phy. Rev. Lett. 102, 038302 (2009). 